\title{
Expert System for topological remedial action discovery in smart grids
}

\author{
A. Marot, B. Donnot, S. Tazi, P. Panciatici (RTE R\&D)
}

\begin{abstract}
For power grid congestion management, lots of research have focused on using generation redispatching, load shedding or demand side management flexibilities. However, a less costly option would be grid topology reconfiguration. Branch switching has been previously explored, since it could be formulated as a linear programming optimization problem we can solve, and showed some benefits. This can further be extended to the broader class of non-linear nodal reconfigurations at substations. In this paper, we present an expert system to automatically discover such topological remedial actions on congested grid states. It comes with a new adapted grid representation, conditional to the congestions of interest, which can be interpreted by operators. To test our expert system, we independently run it on thousands of realistic congested French grid states from 2012 to 2014 with a remedial action discovery success rate of $75 \%$. Our exploration is quite efficient, since it is limited to single substation topological action and is usually successful on first try, even in the case of overloads above $30 \%$.
\end{abstract}

\section{INTRODUCTION}

In the era of smart grids, new flexibilities are needed for congestion management to handle new power flow dynamics without expanding the grid with heavy investments. Controlling injections seem the most intuitive way to deal with such congestions and has been widely explored [1], [2]. Nevertheless, it imposes some constraints on external actors such as producers to gain grid flexibility, which comes at a cost. But power flows are also determined and influenced by the grid topology. For TSOs who own and operate their grid, topology can be changed at negligible cost. But those changes are often highly non-linear and need more advanced algorithms to be controlled. Branch switching [3] is a first step in that direction. However it is only a percent of what could be done if considering a more general class of topology reconfiguration: nodal reconfiguration at substations. New remedial actions or more robust ones could be implemented. In communication networks, topology switching at high frequencies is a crucial aspect of information routing. In power grids however, we have been more cautious with such actions given the hazardous nature of electricity. Indeed there is an associated risk of short circuit that can be harmful or damaging for assets which has to be considered. Nevertheless, some TSOs such as RTE, have been successfully using a fraction of possible topological remedial actions for years, thanks to operator studies and proper asset management.

In this paper, we are interested into curative topological actions. Our goal is to automatically offer our operators many efficient options to manage a stressed grid in a strategic manner, avoiding them the search of such options iteratively in a study as it is today. Our operators know some efficient ones by experience after many years of studies. But their breadth of search has been limited, and their understanding is sometimes flawed by habits. Our expert system should assist them in understanding their problem under study in a specific context, and ultimately help them discover and use more strategical options. Indeed, it should :

- dynamically provide them on new situations a focused representation of the grid, conditioned on their problem.

- suggest them some initial solutions (single topological action) if they exist within that representation.

- allow them to go beyond the machine proposal and find more complex remedial actions based on this representation.

Even in the case no toplogical remedial action is found, they can indeed interpret the visual representation to apprehend quickly the situation and the limits of this expert system, further guiding the search with more expertise.

This philosophy of collaborative human-machine interactions is actually a driver of a larger RTE R\&D project, named Apogee, whose ambition is to build a personal assistant for our control room operators. Related to this work, previous work [4] have investigated labeling of historical operator preventive actions to learn from them. Unfortunately, few contingencies occur in reality. Hence not every curative actions can be observed. Currative actions that were not implemented cannot be learned in the same fashion, which justifies the need for this complementary avenue. Those two approaches however both rely on a same foundation: a counterfactual approach to replay realistic scenarios to learn from, which uses detailed power system simulators at our disposal on top of historical operational data.

The paper is organized as followed. Section II is dedicated to the method, where we describe our counterfactual approach coupled to some expert knowledge to rank a priori the most efficient topological actions. Section III illustrates our method on a didactic example: the IEEE14 case. Section IV further dives into the analysis of topological sensitivities. Section V eventually provides systematic results when running our expert system on the French Power Grid, measuring its performance. Section VI gives conclusions.

\section{METHOD}

\section{A. Problem statement: Congested powerflow}

In this section, we suppose that we have at our disposal a simulator Sim which, given injections vector and a reference topology $\left(P b u s, T o p o^{n}\right)$, compute the power flows $P f^{n}$ on $\mathrm{n}$ lines in service, and detect k overloaded lines $O v^{n}$ :

$$
\left(O v^{n}, P f^{n}\right)=\operatorname{Sim}\left(\text { Pbus }, \text { Topo }^{n}\right)
$$

Those overloads create congestions that grid operators have to manage to ensure grid security. They indeed look 
for topological actions $\mathrm{t}$ at substation $\mathrm{b}\left(a_{b}^{t} \in A_{T o p o^{n}}\right)$, changing the topology from its reference $T o p o^{n}$, to relieve the $\mathrm{k}$ detected overloads such that $O v^{a_{b}^{t}}=0$ :

$$
\left(O v^{a_{b}^{t}}, P f^{a_{b}^{t}}\right)=\operatorname{Sim}\left(P b u s, \text { Topo }^{n} \odot a_{b}^{t}\right)
$$

In other words, we would like to route the flow of those congested paths towards other parallel electrical paths. In a meshed grid, we know they exist, since flows we observe are actually a superposition of flows, shared over multiple paths. But how can we detect them as we are only observing a resulting grid state of entangled flow superposition?

When interested in a specific variable, a proper way to discover what influences it is to directly intervene on it. Interventions have proven very useful in the field of causality [5]. In our case, "what if" our overloaded line was not available? If power could not flow through it, where would it flow? This virtual flow distribution will actually unveil those latent mutually interacting parallel path with our power flows of interest, helping us identifying interesting topological spots to influence our congested flow. With a simulator at our disposal, let's hence run this counterfactual reasoning through a topological sensitivity analysis by switching off our overloads Of $f\left(O v^{n}\right)$ leading to n-k active lines:

$$
\begin{aligned}
& \left(O v^{n-k}, P f^{n-k}\right)=\operatorname{Sim}\left(P b u s, \text { Topo }^{n} \odot \text { Off }\left(O v^{n}\right)\right) \\
& \left(\Delta O v^{n-k}, \Delta P f^{n-k}\right)=\left(O v^{n-k}, P f^{n-k}\right)-\left(O v^{n}, P f^{n}\right)
\end{aligned}
$$

\section{B. Overload distribution Graph: a congested influence zone}

On top of this sensitivity results, we can now build a new representation of the grid, the "Overload distribution Graph" $\left(G_{O v}\right)$. It is similar to the usual representation of a directed power flow graph $G_{P f}$ over a grid, with the same connectivity and directions, but whose edge weights are $\Delta P f^{n-k}$ instead of $P f^{n}$ (see Figure 1). However it is rather different as it is not a global graph over the grid, but it becomes a local influent zone when only considering sensitive flow distributions over a threshold th $(t h=5 \%$ for French Power Grid), below which we truncate our $G_{O v}$.
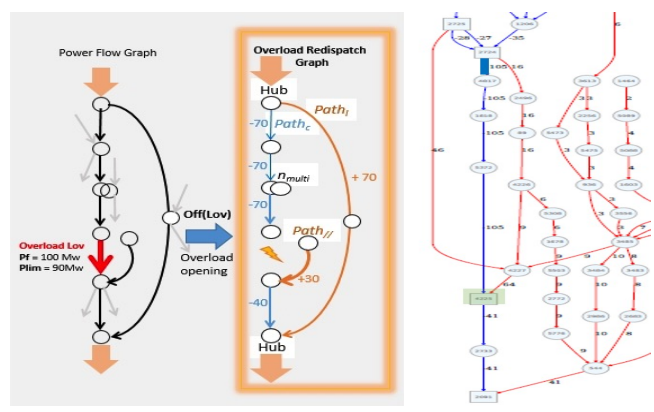

Fig. 1. On the left, two local grid representations around the overload: a zoomed $G_{P f}$ and the $G_{O v}$. On $G_{P f}$, grey flows are insensitive ones to $\operatorname{Off}\left(O v^{n}\right)$ and do not appear on $G_{O v}$. On $G_{O v}$, blue lines have decreasing flow while red lines have increasing flow. Expert labels are represented. On the right, a real $G_{O v}$ example on case 6515rte. Congested line $l_{4815}$ in dark blue and remedial substation Bus ${ }_{4225}$ in green.
From our $G_{O v}$, which defines a relevant influence zone to explore, we want to identify topological spots to route our congested flow. To do so, we first extract some meaningful structure from the graph as on Figure 1 to reason on:

- the constrained Path , that is the connected flow path to the overload with negative distribution.

- upstream $U a$ and downstream $D a$ areas relative to the overload given the initial flow direction on $G_{P f}$.

- Parallel $P a t h / /$ with positive distribution, paths that supply similar loads downstream than $P a t h_{c}$.

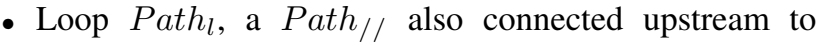
$P a t h_{c}$ : flows are also supplied by similar productions.

- local routing spots: $H u b$ as nodes intersecting $P a t h_{c}$ and $P a t h / /$ or $P a t h_{l}$, multi node substations $n_{m u l t i}$ and Open circuit lines $L_{o c}$.

Based on the detection of such structural elements, we can further identify 4 different $G_{O v}$ cases we can encounter:

- Looped $G_{O v}$ if we have at least one $P a t h_{l}$

- Parallel $G_{O v}$ if we have at least one $P a t h / /$

- Multi Nodes $G_{O v}$ if we have at least one $n_{m u l t i}$

- Unmeshed $G_{O v}$ if there is no meshing option.

\section{Expert knowledge: identifying routing buses}

Let's now introduce some expert knowledge to rank the topological actions that could help us route the flow differently. It relies on two expert principles, that is modifying the relative impedance of electrical paths or creating a new injection pathway between 2 zones at different phase potentials. More specifically:

1) $H u b$ are the most interesting spots because you can locally route flow by splitting nodes and pushing the incoming or outgoing injections towards $P a t h / /$, connecting them together, while isolating from those injections our $P a t h_{c}$, and as a result from our overload.

2) On $\mathrm{Path}_{c}$ we would like to increase its impedance to hinder the flow by node splitting or branch switching.

3) On $\mathrm{Path}_{/ /}$we would like to decrease its impedance to ease the flow. To do so we can merge $n_{\text {multi }}$ or switch on $L_{o c}$.

4) Over Da on $P a t h_{c}$, we might want to merge $n_{m u l t i}$ or switch on $L_{o c}$ as well but with the intent of bringing power from elsewhere. Looking at nodal phase potentials, we can guess in which direction power will flow when merged. This tells us if it should be beneficial or not. Conversely for $U a$ looking for loads.

\section{Ranking topologies with Expert knowledge}

We eventually assess by simulation topologies at substations ranked along these categories 1 to 4 . When two substations belong to the same category, we prioritize the one with the most ingoing or outgoing, negative or positive, flow distribution on $G_{O v}$. In the case of Unmeshed $G_{O v}$, topology is inefficient, the only solution will be load shedding: we can hence detect infeasible cases.

Finally, for a given substation, we also rank topologies, as on Figure 2, since there are very often more than 20 possible 
configurations with the same active lines. The main goal is to "break" Path , to increase this path impedance, setting ingoing and outgoing $P a t h_{c}$ on 2 different nodes. Second, you want to route as much flow as possible on $\mathrm{Path}_{/ /}$. Given those two principles, if you are on $U a$, you want to connect on one node outgoing $\mathrm{Path}_{c}$ towards the overload to non-sensitive ingoing and outgoing flows, plus local loads. On another node you connect the remaining sensitive flows, ingoing $\mathrm{Path}_{c}, \mathrm{Path}_{/ /}$, and production. Conversely on Da.

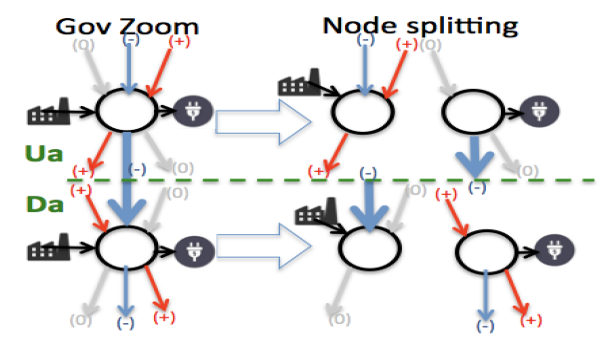

Fig. 2. How to preferably reconfigure electrical nodes in $U a$ or $D a$, given the related $G_{O v}$ elements for a given overload (bold blue), plus the local productions and loads as well as the non-sensitive grey path.

Our overall expert system is finally depicted on Figure 3.

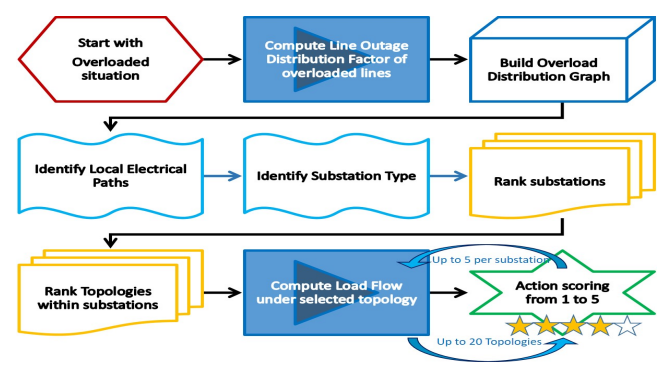

Fig. 3. Expert System Algorithm for topological curative action discovery.

\section{A DIDACTIC EXAMPLE}

\section{A. The IEEE14 case}

For illustration, we will consider the IEEE 14 power system and apply our algorithm to it. Considering line 10 connecting bus 5 to 6 , an interconnection between the high voltage and low voltage grids, we can imagine it getting overloaded when load demand is high. We will hence study how to reroute part of this powerflow from our reference meshed topology as on Figure 4. While changing topology, we don't want any line being turned off as it is often more robust to operate all of them to increase grid's capacity.

\section{B. Overload Distribution Graph over line $l_{5} \rightarrow 6$}

From Figure 4, we can observe our influence graph after opening $l_{5 \rightarrow 6}$ : this is a Looped $G_{O v}$ we computed through Matpower [6]. The flow distribution highlights a zone of topological influence to consider, mainly the low voltage grid, while discarding the high voltage grid. More precisely, buses being discarded at this stage are: $\{1,2,3,12\}$. In terms of structure on the graph, we identify several paths:
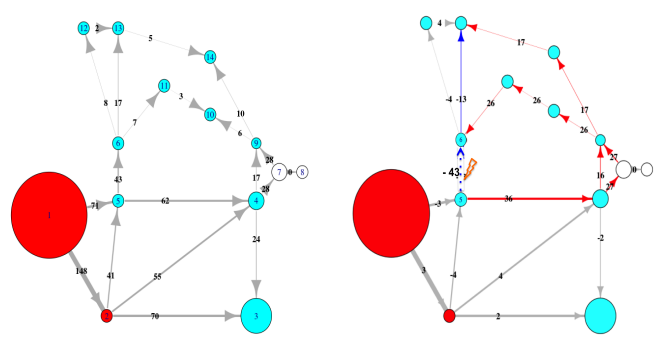

Fig. 4. IEEE14 system and its power flow on the left. Red nodes for production and blue for loads. On the right, our $G_{10}$, the overload distribution graph for our powerflow of $P f_{5} \rightarrow 6$. Red edges for flow increase, blue for flow decrease and grey for non-sensitive flows.

- Path $_{c}=5 \rightarrow 6 \rightarrow 13(U a=\{5\}$ and $D a=\{6,13\})$

- Path $_{l 1}=5 \rightarrow \ldots \rightarrow 9 \rightarrow \ldots \rightarrow 6$

- Path $_{l 2}=5 \rightarrow \ldots \rightarrow 9 \rightarrow \ldots \rightarrow 13$

- $H u b s=\{5,6,13\}$

Over the $P a t h_{l}$, we can further discard any substation that is not a hub and whose topology is fully connected as a single electrical node, that are substations $\{4,7,8,9,10,11,14\}$. Indeed, as explained in Section II) C), while we would like to push more flow over such paths, we can only perform here a node splitting operation for those nodes, which will increase the path impedance and hence repel flows. Hence it will not ease the flow on $l_{5 \rightarrow 6}$ but load it even more.

\section{Topology reconfiguration at Bus 5 and 6 as remedial actions}

We are now left with buses on the constrained path here, especially our $H u b s$, to solve our problem. Bus 6 is the most promising and ranked first as in section II) D), since it is a hub in the middle of $P a t h_{c}$, which we can "break" while still supplying the loads from a parallel path, and it has a high ingoing distribution flow (45 MW). Being in $D a$, we perform the following node splitting on $\mathrm{Bus}_{6}$ (Figure 2) :

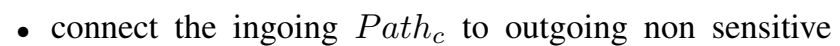
path and to local productions or ingoing flows, that is Node $1=\{10,12\}$

- connect the ingoing $P a t h_{l}$ to outgoing $P a t h_{c}$, and local loads. that is Node $2=\left\{11,13, \operatorname{load}_{6}\right\}$

This indeed results in a $15 \mathrm{MW}$ decrease, corresponding to $30 \%$ of the initial power flow, which is quite effective. Of course, you should avoid making new congestions and monitor the powerflow on other loaded lines. load $_{6}$ could be switched to node 1 here as an alternative topology for a smoother flow distribution, resulting in a $4 \mathrm{MW}$ decrease.

For the two remaining $\mathrm{Hubs,Bus_{5 }}$ is better suited than $B u s_{13}$ since about $85 \%$ of our overload fictively got redispatched there based on $G_{O v}$, compared to $40 \%$ at $B u s_{13}$ after intervening on $l_{5} \rightarrow 6$. Not to mention that $B u s_{13}$ cannot be split into 2 nodes here while preserving a meshed grid, having only 3 lines and not a minimum of 4 . Being in $U a$, we should perform the following node splitting on $\mathrm{Bus}_{5}$ :

- connect the outgoing $P a t h_{c}$ to outgoing non influential path and local loads, and to minimum ingoing injection 
paths, that is $N o d e 1=\left\{5,10, \operatorname{load}_{5}\right\}$

- connect the outgoing $\mathrm{Path}_{l}$ to local productions and to maximum ingoing injection paths, so $\operatorname{Node} 2=\{2,7\}$

This indeed results in a $2.5 \mathrm{MW}$ or $6 \%$ decrease. Another option could be connecting all outgoing paths on Node1 $=$ $\left\{5,7, \operatorname{load}_{5}\right\}$ and all ingoing paths on Node $2=\{2,5\}$ resulting in a $6 \mathrm{MW}$ decrease as on Figure 5 but it is more brutal as it changes the topology mesh and flow direction.
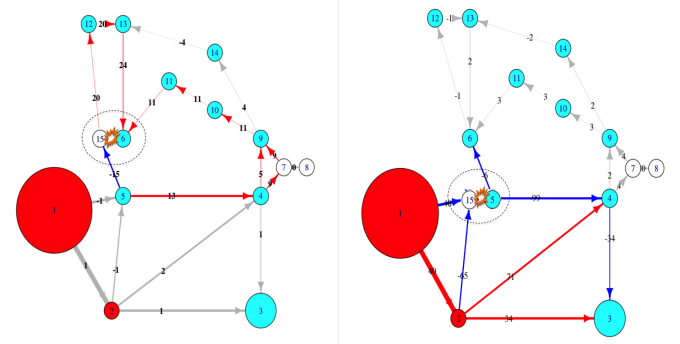

Fig. 5. Topology reconfiguration on IEEE14 at identified Hubs, Bus $s_{5}$ and $B u s_{6}$. On the left, the flow distribution after $B u s_{6}$ most promising node splitting. On the left, the flow distribution after Bus $s_{5}$ node splitting.

Topological reconfigurations at buses 5 and 6 happen to be potential remedial actions when line 10 gets overloaded. They are actually the only topological ones that keeps a meshed grid. We knew it a priori based on our expert system without any greedy search. Of course, this could be found by other methods on such a small example. But our method can hopefully scale to much larger grids, such as RTE French power grid with about 6000 buses, 500 of which having more than 7 connected power lines making the meshing more complex and the search space much bigger. If our method seems already effective, can we rank the topologies more formally, and interpret those results more globally?

\section{ANALYSIS OF TOPOLOGICAL SENSITIVITIES}

\section{A. From local expertise to global and formal analysis}

Beyond this expertize which helps prioritizing buses to look at qualitatively, we could investigate some theoretical foundation for it. Doing so, it could be possible to use more global quantitative measures to better rank the topologies.

We are interested into influencing power flows, which are mainly driven by active power. The DC approximation is often good enough to screen the space of flexibilities at our disposal with about $5 \%$ accuracy loss. Given Bbus, the Laplacian adjacency matrix, $\theta$, the node potentials, and $x$, the line impedances, we have the following load flow equations:

$$
\begin{gathered}
\text { Pbus }=\text { Bbus } \times \theta+\text { Pbus }_{\text {shift }} \\
P f_{i j}=\frac{1}{x_{i j}}\left(\theta_{i}-\theta_{j}-\theta_{\text {shift }}\right)
\end{gathered}
$$

Bbus being a Laplacian, we can compute its pseudoinverse Bbus $^{+}($Topo $)=\left[F_{1}^{\text {bus }}|\ldots| F_{m}^{b u s}\right]$. Bbus and $\mathrm{Bbus}^{+}$only depend on the topology Topo. Once computed, we can actually get at every bus $\mathrm{b}$ the contribution factor $F_{b}^{b u s}$ of every Pbus to the potential $\theta_{b}$.

$$
\theta_{b}=F_{b}^{b u s} \times\left(\text { Pbus }- \text { Pbus } s_{\text {shift }}\right)
$$

Putting it into equation (6), and introducing Pbus $_{\text {shifted }}=$ Pbus - Pbus $_{\text {shift }}$, we have:

$$
\begin{aligned}
P f_{i j} & =\frac{1}{x_{i j}}\left(F_{i}^{b u s}-F_{j}^{b u s}\right) \times P b u s_{\text {shifted }} \\
& =P T D F_{i j} \times \text { Pbus }_{\text {shifted }}
\end{aligned}
$$

Here appears the well-known Power Transfer Distribution Factors (PTDF) used to study power flow sensitivity to injections. In our case, we are interested into Topology Distribution Factors, an extension of Line Outage Distribution Factors (LODF). We can further get the contribution $C_{i j}^{b u s}$ of every Pbus to each powerflow $P f_{i j}$ on this grid state:

$$
P f_{i j}=\sum_{b=1}^{m} P T D F_{i j}^{b} * \text { Pbus }_{\text {shifted }}^{b}=\sum_{b=1}^{m} C_{i j}^{b}
$$

For a given topology $T o p o^{n}$, we can hence detect the most influent $P b u s_{k}$ on $P f_{i j}$ given their related contribution $C_{i j}^{b}$. By selectively analyzing the evolution of those specific sensible influences $\left(\right.$ bus $_{b^{\prime}} \in S\left(P f_{i j}\right)$ ) under $a_{b}^{t}$, and discarding others, we can get an estimate on how much our powerflow of interest changes, to anticipate $a_{b}^{t}$,s effectiveness a priori:

$S\left(P f_{i j}\right)=\left\{\operatorname{argmin}_{S^{\prime} \subseteq B \text { uses }}\left|S^{\prime}\right| \mid \frac{\left|P f_{i j}-\sum_{\left.b^{\prime} \in S^{\prime}\right)} C_{i j}^{b^{\prime}, n}\right|}{P f_{i j}}<=10 \%\right\}$

$$
\left\{\begin{aligned}
\Delta P f_{i j}^{a_{b}^{t}} & =\sum_{b^{\prime}=1}^{m} C_{i j}^{b^{\prime}, a_{b}^{t}}-\sum_{b^{\prime}=1}^{m} C_{i j}^{b^{\prime}, n} \\
& \approx \sum_{b^{\prime} \in S\left(P f_{i j}\right)}\left(P T D F_{i j}^{b^{\prime}, a_{b}^{t}}-P T D F_{i j}^{b^{\prime}, n}\right) P b u s_{\text {shifted }}^{b^{\prime}}
\end{aligned}\right.
$$

Moreover, we can interpret more globally, and not only in terms of local flows, as we did based on our $G_{O v}$, how long distance injection influence evolves to explain a change in powerflow. This could be further understood as a relative change in effective resistance Req, between our overload and those influent injections, to highlight which path impedance really changed. Indeed $B b u s^{+}$and $R e q$ are closely related:

$$
\operatorname{Req}_{i j}=\mathrm{Bbus}_{i i}^{+}+\text {Bbus }_{j j}^{+}-2 B b u s_{i j}^{+}
$$

In terms of computation, Bbus ${ }^{+}\left(\right.$Topo $\left.^{n} \odot a_{b}^{t}\right)$, and further PTDF, coefficient can be computed efficiently, incrementally and with parallelism, from original $\mathrm{Bbus}^{+}\left(\right.$Topo $\left.^{n}\right)$ under a topological change [7]. Authors in [8] give additional insights for interpretation by detecting flow cycles while defining a dual representation of the network.

\section{B. IEEE14 case: topology sensitivity interpretation}

Based on this derivation, let's compute our injection contributions on our IEEE14 example to interpret more deeply what happened under our influential topology changes. On Figure 6, one can see the contribution evolution of every 


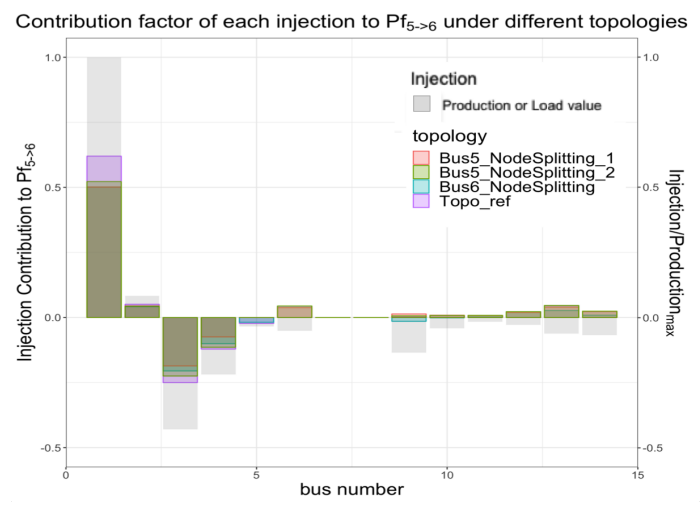

Fig. 6. Evolution of injection influence to $P f_{56}$ under different topologies in colors, relatively to injection values in grey, to interpret topology impact.

injection to our powerflow $P f_{56} . P f_{56}$ is mostly driven by the main production at bus 1 , the loads at buses $6,12,13,14$ in the West consumption area. It also feels the loads at buses 3 , 4 and 5 which rather pull the flow in the opposite direction, masking some of the influence of production 1. However, our powerflow does not feel the loads in the East Part of the grid because East and West are actually balanced, with similary meshed subgrids to supply them. In this case, we have $S\left(P f_{56}\right)=\{1,3,4,5,6,12,13,14\}$

When we change the topology at $B u s_{6}$, the West Part Load influence decreases and $B u s_{9}$ influence becomes sensible. Indeed, the electrical paths through $B_{u s} s_{6}$ became longer with greater impedance, giving more importance to $\mathrm{Bus}_{9}$ to now supply the loads all over the distribution grid, breaking the subtle subgrid balance. Contributions of production 1 and loads 3 and 4 diminishes consequently but proportionally. As a results we estimate $\Delta P f_{56}^{a_{b u s}^{1} 6} \approx-10.8 M W$.

When we change topology at Bus $_{5}$ in the second configuration, we make our line relatively closer to loads 3 and 4 and further from production 1 compared to previously. This pulls the flow in the opposite direction, hence decreasing it. This is even more true in the second node splitting configuration at bus 5 for which production 1 gets far away, leading to $\Delta P f_{56}^{a_{b u s}^{1}} \approx-3.0 M W$ and $\Delta P f_{56}^{a_{b u s 5}^{2}} \approx-5.6 M W$.

Even if our estimates are rough approximations on such a small grid, we can rank them a priori in the right order. Especially at $\mathrm{Bus}_{5}$ where it was unclear which configuration will be best a priori with only an understanding of interactions locally at this bus over our $G_{O v}$ on Figure 4.

\section{Results on French Power Grid}

We will now describe results on the larger grid of interest to us: the French Power Grid. We will present one more reproducible and relativeley difficult example over the Matpower 6515 rtecase. Finally, we will share systematic results of our expert system, after running it over thousands of situations, and discuss them.

\section{A. One More Example: the 6515 RTE case}

Four historical French Power Grid snapshots have been recently released [9]. Running through congested N-1 situ- ations over those cases, we looked for illustrative ones for our method. One was the following: on case 6515 , after a contingency on $l_{4816}, l_{4815}$ gets overloaded when setting the thermal limit to its $95 \mathrm{MW}$ value. The related Looped $G_{O v}$ can be seen on Figure 1. The influence zone is quite large but we can extract from it our structural elements $P a t h_{c}$, $P a t h_{l}$ and $H u b$ to guide our search:

- Path $_{l 1}=2541 \rightarrow \ldots \rightarrow 3947$

- Path $_{l 2}=2540 \rightarrow \ldots \rightarrow 3947$

- $H u b s=\{2540,2541,3947\}$

Hub 3947 is the most promising, belonging to 2 looped paths. And indeed, there is a topological remedial action that has really been implemented on the grid in the past, with 2 electrical nodes leading to $12 \mathrm{MW}$ decrease and resulting in a flow of $92 \mathrm{MW}$. Even if the bus is 3 hops away from our overload, and there can be many other buses to consider at this distance, it is the first choice of our expert system.It actually appears that is the only relevant one beside opening some lines. We here illustrated the expert system effectiveness at being selective even on a larger zone, proving to be really helpful in the remedial action search.

\section{B. Systematic results on thousands of realistic cases}

To test our expert system systematically on a larger grid, we had to generate a realistic database of congested situations based on French historical snapshots between 2012 and 2014, given that we rarely observe any overloads on real snapshots. To be representative, we selected $9 \mathrm{am}$, noon, $4 \mathrm{pm}, 7 \mathrm{pm}$ snapshots over the days, on which we run security analysis. We then studied the overloaded situations, with at least $2 \%$ overload, that could have occurred after a contingency. On average, there are 80 risky contingencies per snapshot to study, over 10.000 power lines.

To consider realistic topological actions, we restricted ourselves to the ones that have been applied at least once in a snapshot in the past. This makes 52.539 possible topologies over 6.091 substations. Our heuristic will only pick up to 20 topologies among these, up to 5 per substation, and try to find as many remedial actions as possible. We also tested up to 3 branch switching on $\mathrm{Path}_{c}$ per congested situation.

Scores from 1 to 5 are given to an action: 5 if every overloads disappeared, 4 if an overload disappeared without stressing the network, 3 if at least $30 \%$ of an overload was relieved, 2 if an overload was relieved but an other appeared or got worse, 1 as failed if no overloads were alleviated or if it resulted in some load shedding or production distribution.

From the result table on Figure 7, we see that most of the feasible situations are Looped ones, meaning meshed local grids. In these situations, individual topological curative actions can be quite effective, with an overall success rate (score above 4) of $76 \%$ to relieve overloads.

Independently analyzing branch switching and nodal reconfiguration, switching leads to a remedial action $55 \%$ of the time but if not, worsen the situation (score below 2) in $39 \%$, while nodal reconfiguration helps in $64 \%$ cases with only $19 \%$ worsened situations from the result table on Figure 8. For Extra High Voltage overloaded lines (30\% of 


\begin{tabular}{|l|r|rrrrr|}
\hline \multirow{2}{*}{ Gov Type } & \multicolumn{5}{|c|}{ success score rate } & number \\
\cline { 2 - 7 } & 1 & 2 & 3 & 4 & 5 & of cases \\
\hline Unmeshed & 97 & 0 & 0 & 0 & 3 & 29076 \\
\hline Multi Nodes & 36 & 2 & 5 & 9 & 48 & 11340 \\
\hline Looped & 0 & 14 & 10 & 24 & 52 & 40886 \\
\hline Parallel & 75 & 3 & 1 & 5 & 16 & 23220 \\
\hline
\end{tabular}

Fig. 7. Summary table of most efficient topological action found per congested situation (max 20 topologies explored over 5 substations) by our expert system on French Power Grid.

cases), success rate even reach $79 \%$ for nodal reconfiguration while branch switching remains at $57 \%$. Finally, for VHV lines in cases of high overloads (above $30 \%$ of their thermal limit), we still manage to achieve a high success rate of $73 \%$ with nodal reconfiguration. This demonstrates that our expert system can be very effective at discovering single topological actions for meshed high voltage transmission grids. A control room operator can further implement such suggestions on a congested situation or compose more complex topology reconfiguration from those unitary results.

\begin{tabular}{l|rrrrr|r|}
\hline \multirow{2}{*}{ Type } & \multicolumn{5}{|c|}{ success score rate } & number \\
\cline { 2 - 8 } & 1 & 2 & 3 & 4 & 5 & of cases \\
\hline All Types & 0 & 14 & 10 & 24 & 52 & 40886 \\
\hline Branch switching (BS) & 0 & 39 & 6 & 19 & 36 & 40782 \\
\hline nodal reconfiration (NR) & 10 & 9 & 17 & 18 & 46 & 39988 \\
\hline Very High Voltage (BS) & 0 & 35 & 8 & 16 & 41 & 16433 \\
\hline Very High Voltage (NR) & 4 & 5 & 12 & 20 & 59 & 16397 \\
\hline VHV and overoad>=30\% (NR) & 1 & 17 & 10 & 20 & 53 & 865 \\
\hline
\end{tabular}

Fig. 8. Summary Table of most efficient topological action in Looped congested situations, depending on the type of topological action and the nature of the overload.

Finally, looking at the relevance of nodal topology reconfiguration ranking overall on Figure 9 bar plots, we notice that, when we find a solution for a meshed and complex situation (at least 10 actions tested on a congested situation), we can find a successful one for $70 \%$ of cases when testing up to 3 actions. The chances of success per action tested then decrease steadily with a deeper search. In terms of computation, it requires only 3 load-flows (which can be computed in parallel) to assess the relevance of these actions, in addition to a prior load-flow to build our $G_{O v}$. The graph analysis computation on $G_{O v}$ is negligible. When looking closer at the substation ranking, we also notice a higher chance of success for the first chosen substation, above $50 \%$, and a steady decrease to $20 \%$ chance for the 5 th substation, when at least 3 substations have been explored. These results indicates that our expert system properly ranks the remedial actions a priori. Hence this demonstrates a good exploration strategy. This ranking could be further improved when coupled with distribution factor computations as defined in section IV) A), or with an existing remedial action database.

\section{Building knowledge on grid topology flexibility}

Our expert system when run over many historical situations can automatically initialize a database of topological remedial actions. In our systematic study, it discovered 29208 different remedial actions (different efficient action for each overloaded lines), over 2098 overloaded lines with
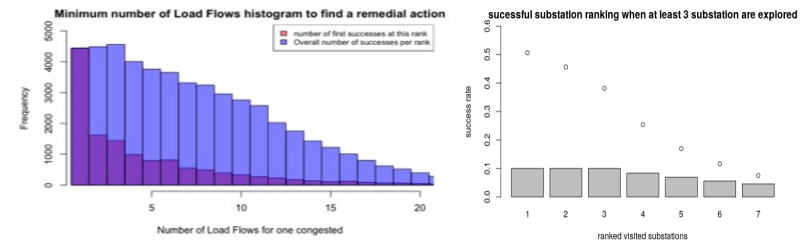

Fig. 9. On the left, histograms of the minimum load flow required to find a topological solution in congested situations in red, and of the number of successes per topology ranking in blue. On the right, success rate at a substation according to its ranking a priori, when at least 3 substations have been explored, summing to 14546 situations. Grey histogram showing the sample proportions of situations for a given substation exploration depth

a median of 8 remedial actions per line. These numbers could be further increased if considering actions that have not been implemented. It could hence highlight interesting nodal reconfiguration that were not considered, pushing forward the need for studying such flexibilities and maybe upgrading substation assets to enable its implementation.

\section{CONCLUSIONS}

Our expert knowledge system proved to be successful by discovering topological remedial actions a priori, beyond branch switching, in a selective manner, relying on a simple local counterfactual reasoning and few load-flow computations. The underlying "distribution graph" can further be interpreted by a control room operator to discover more complex remedial actions. This successful counterfactual reasoning can also be jointly applied to the whole grid to represent overall zonal segmentation of it [10]. In terms of applications, our expert system can be used in real time to make remedial action suggestions or can help initialize a remedial action database given historical snapshots. Systematic results on RTE's French Transmission grid when applying our method highlighted the potential of topological actions to make smarter grids, making the point that such flexibilities deserve more research and studies in the future.

\section{REFERENCES}

[1] Kothari D. Pillay A., Karthikeyan S. Congestion management in power systems a review. International Journal of Electrical Power and Energy Systems, 2015.

[2] Djokic S. Gunda J., Harrison G. Remedial actions for security constraint management of overstressed power systems. IEEE Transactions on Power Systems, 2018.

[3] Ferris M. Fisher E., O’Neill R. Optimal branch switching. IEEE Transactions on Power Systems, 2008.

[4] I. Guyon B. Donnot, A. Marot and al. Introducing machine learning for power system operation support. IEEE IREP conference, 2017.

[5] J. Pearl. Causality. Cambridge university press, 2009.

[6] Matpower:steady-state operations, planning, and analysis tools for power systems research and education.

[7] Zhang Z.-L. Ranjan G. and Boley D. Incremental computation of pseudo-inverse of laplacian. Combinatorial Optimization and Applications, Springer, 2014.

[8] A dual method for computing power transfer distribution factors.

[9] J. Maeght C. Josz, S. Fliscounakis and P. Panciatici. Ac power flow data in matpower and qcqp format: itesla, rte snapshots, and pegase. ArXiv e-printsarXiv:1603.01533, 2016

[10] A. Marot, S. Tazi, B. Donnot, and P. Panciatici. Guided machine learning for power grid segmentation. IEEE ISGT Europe, 2018. 